\title{
Coexistence of Triplet Superconductivity and Spin Density Wave
}

\author{
Wei Zhang and C. A. R. Sá de Melo \\ School of Physics, Georgia Institute of Technology, Atlanta, Georgia 30332
}

(Dated: December 16, 2018)

\begin{abstract}
We discuss the possibility of coexistence of spin density wave (antiferromagnetism) and triplet superconductivity as a particular example of a broad class of systems where the interplay of magnetism and superconductivity is important. We focus on the case of quasi-one-dimensional metals, where it is known that antiferromagnetism is in close proximity to triplet superconductivity in the pressure versus temperature phase diagram. Over a range of pressures, we propose an intermediate non-uniform phase consisting of antiferromagnetic and triplet superconducting orders. In the coexistence region, we propose a flop transition in the spin density wave order parameter vector, which affects the nature of the superconducting state.
\end{abstract}

PACS numbers: 74.70.Kn, 74.25.DW 
The competition or coexistence of magnetic order and superconductivity is a very important problem in condensed matter physics. There is a broad class of systems that present magnetic order and superconductivity in close vicinity. One of the most important systems are the Copper oxides, where singlet superconductivity is found next to antiferromagnetism [1]. Another interesting system is Strontium Ruthenate $\mathrm{Sr}_{2} \mathrm{RuO}_{4}$, where the proximity to ferromagnetism has been argued as being important to the existence of possible triplet superconductivity in these materials [2]. Furthermore the ferromagnetic superconductors $\mathrm{ZrZn}_{2}$ and $\mathrm{UGe}_{2}$ have stimulated a debate on the coexistence of ferromagnetism and triplet or singlet superconductivity [3, 4]. However, unlike any of these previous examples, we discuss in this manuscript a quasi-one-dimensional organic superconductor, the Bechgaard salt $(\mathrm{TMTSF})_{2} \mathrm{PF}_{6}$, which has a phase diagram of neighboring antiferromagnetism (AFM) and triplet superconductivity (TSC) [5].

The antiferromagnetic state of (TMTSF $)_{2} \mathrm{PF}_{6}$ is present at temperatures $T<12 \mathrm{~K}$ and pressures $P<6 \mathrm{kbar}$, and is characterized by a spin density wave (SDW) [5]. The SDW order parameter $\mathbf{N}$ (Neel vector) is anisotropic, having an easy axis along the crystallographic $\mathbf{b}^{\prime}$ axis [6], which is also the intermediate direction for conductivity. This antiferromagnetic state is supressed at pressures higher than 6 kbar, where a superconducting instability takes over at low temperatures $\left(T<T_{c} \approx 1.2 \mathrm{~K}\right)$. This superconducting state is very likely to be triplet, as suggested by upper critical fields [7] and NMR 8] measurements. Recent experiments [9, 10, 11] suggest a region of macroscopic coexistence between TSC and SDW, where both orders are non-uniform. This coexistence region can be related to existing theoretical proposals. For instance, strictly one-dimensional theories invoking SO(4) symmetry [12], or negative interface energies [13] have allowed for coexisting TSC and SDW. However, these previous theories are not directly applicable to three-dimensional but highly anisotropic superconductors like the Bechgaard salts, where the $\mathrm{SO}(4)$ symmetry is absent, and negative interface energies are not necessary conditions for the coexistence. In this manuscript, we derive microscopically the pressure versus temperature phase diagram indicating the TSC, the SDW and the TSC/SDW phases, and show that the TSC and SDW order parameters are both non-uniform in the coexistence region. Furthermore, we investigate the effects of an external magnetic field and suggest that a canting transition of the SDW order parameter may occur, and alter the nature of the TSC state in the coexistence region. 
The compound (TMTSF) ${ }_{2} \mathrm{PF}_{6}$ can be described approximately by an orthorhombic lattice with dispersion

$$
\epsilon_{\mathbf{k}}=-\left|t_{x}\right| \cos \left(k_{x} a\right)-\left|t_{y}\right| \cos \left(k_{y} b\right)-\left|t_{z}\right| \cos \left(k_{z} c\right)
$$

where transfer integrals $\left|t_{x}\right|,\left|t_{y}\right|$ and $\left|t_{z}\right|$ satisfy the relations $\left|t_{x}\right| \gg\left|t_{y}\right| \gg\left|t_{z}\right|$ representing the quasi-one-dimensionality. Here, $a, b$, and $c$ correspond to unit cell lengths along the crystallographic axes $\mathbf{a}(\mathrm{x}), \mathbf{b}^{\prime}(\mathrm{y})$ and $\mathbf{c}^{*}(\mathrm{z})$, respectively.

We use natural units $\left(\hbar=k_{B}=c=1\right)$ and work with Hamiltonian $\mathcal{H}=\mathcal{H}_{0}+\mathcal{H}_{\text {int }}$, where the non-interacting part is $\mathcal{H}_{0}=\sum_{\mathbf{k}, \alpha} \xi_{\mathbf{k}} c_{\mathbf{k}, \alpha}^{\dagger} c_{\mathbf{k}, \alpha}$, and $\xi_{\mathbf{k}}=\epsilon_{\mathbf{k}}-\mu$ is the dispersion shifted by the chemical potential, which may include a Hartree shift. The interaction part is

$$
\begin{aligned}
\mathcal{H}_{\text {int }} & =\sum_{\mathbf{k} \mathbf{k}^{\prime} \mathbf{p}} \sum_{\alpha \beta \gamma \delta} V\left(\mathbf{k}, \mathbf{k}^{\prime}\right) \mathbf{d}_{\alpha \beta}^{\dagger}(\mathbf{k}, \mathbf{p}) \cdot \mathbf{d}_{\gamma \delta}\left(\mathbf{k}^{\prime}, \mathbf{p}\right) \\
& +\sum_{\mathbf{k k}^{\prime} \mathbf{q}} \sum_{\alpha \beta \gamma \delta} J(\mathbf{q}) \mathbf{s}_{\alpha \beta}^{\dagger}(\mathbf{k}, \mathbf{q}) \cdot \mathbf{s}_{\gamma \delta}\left(\mathbf{k}^{\prime}, \mathbf{q}\right),
\end{aligned}
$$

where the first and second terms describe interactions in TSC and SDW channels, respectively. These interactions allow for the possibility of competition or coexistence of TSC and SDW instabilities at low temperatures. Here, $\alpha, \beta, \gamma$ and $\delta$ are spin indices and $\mathbf{k}, \mathbf{k}^{\prime}, \mathbf{p}$ and $\mathbf{q}$ represent linear momenta. The vector operator $\mathbf{d}_{\alpha \beta}^{\dagger}(\mathbf{k}, \mathbf{p}) \equiv c_{\mathbf{k}+\mathbf{p} / 2, \alpha}^{\dagger} \mathbf{v}_{\alpha \beta} c_{-\mathbf{k}+\mathbf{p} / 2, \beta}^{\dagger}$, and $\mathbf{s}_{\alpha \beta}^{\dagger}(\mathbf{k}, \mathbf{q}) \equiv c_{\mathbf{k}-\mathbf{q} / 2, \alpha}^{\dagger} \boldsymbol{\sigma}_{\alpha \beta} c_{\mathbf{k}+\mathbf{q} / 2, \beta}$. The matrix $\mathbf{v}_{\alpha \beta}=\left(i \boldsymbol{\sigma} \sigma_{y}\right)_{\alpha \beta}$, and $\sigma_{i}$ are Pauli matrices. In the case of weak spin-orbit coupling, the TSC interaction $V\left(\mathbf{k}, \mathbf{k}^{\prime}\right)$ can be choosen as [14]

$$
V\left(\mathbf{k}, \mathbf{k}^{\prime}\right)=V h_{\Gamma}\left(\mathbf{k}, \mathbf{k}^{\prime}\right) \phi_{\Gamma}(\mathbf{k}) \phi_{\Gamma}\left(\mathbf{k}^{\prime}\right)
$$

where $V$ is a prefactor with dimension of energy, $h_{\Gamma}\left(\mathbf{k}, \mathbf{k}^{\prime}\right)\left[\phi_{\Gamma}(\mathbf{k})\right]$ characterizes the momentum dependence [symmetry basis function] for an irreducible representation $\Gamma$ of the orthorhombic group $D_{2 \mathrm{~h}}$. Without loss of the generality regarding symmetry properties, we take $h_{\Gamma}\left(\mathbf{k}, \mathbf{k}^{\prime}\right)=1$, and consider only unitary triplet states corresponding to $p_{x}$ symmetry.

The order parameter for TSC can be defined as

$$
\mathbf{D}(\mathbf{p})=\left\langle\sum_{\mathbf{k}, \alpha \beta} V \phi_{\Gamma}(\mathbf{k}) \mathbf{d}_{\alpha \beta}(\mathbf{k}, \mathbf{p})\right\rangle
$$

while the SDW order parameter can be defined as

$$
\mathbf{N}(\mathbf{q})=J(\mathbf{q})\left\langle\sum_{\mathbf{k}, \alpha \beta} \mathbf{s}_{\alpha \beta}(\mathbf{k}, \mathbf{q})\right\rangle
$$


With these definitions, the effective Hamiltonian is

$$
\mathcal{H}_{\mathrm{eff}}=\mathcal{H}_{0}+\mathcal{H}_{\mathrm{TSC}}+\mathcal{H}_{\mathrm{SDW}}
$$

where the TSC contribution is $\mathcal{H}_{\mathrm{TSC}}=\sum_{\mathbf{p}}\left[\mathbf{D}^{\dagger}(\mathbf{p}) \cdot \sum_{\mathbf{k}, \alpha \beta} \phi_{\Gamma}(\mathbf{k}) \mathbf{d}_{\alpha \beta}(\mathbf{k}, \mathbf{p})+\right.$ H.C. $]-\sum_{\mathbf{p}} \mathbf{D}^{\dagger}(\mathbf{p})$. $\mathbf{D}(\mathbf{p}) / V$, and the TSC term is $\mathcal{H}_{\mathrm{SDW}}=\sum_{\mathbf{q}}\left[\mathbf{N}(-\mathbf{q}) \cdot \sum_{\mathbf{k}, \alpha \beta} \mathbf{S}_{\alpha \beta}(\mathbf{k}, \mathbf{q})+\right.$ H.C. $]-\sum_{\mathbf{q}} \mathbf{N}(-\mathbf{q}) \cdot$ $\mathbf{N}(\mathbf{q}) / J(\mathbf{q})$. The effective action of this Hamiltonian as a function of $\mathbf{D}(\mathbf{p})$ and $\mathbf{N}(\mathbf{q})$ is obtained by integrating out the fermions. The quadratic terms are

$$
\begin{aligned}
S_{2}^{\mathrm{TSC}} & =\sum_{\mathbf{p}} A(\mathbf{p}) \mathbf{D}^{\dagger}(\mathbf{p}) \cdot \mathbf{D}(\mathbf{p}), \\
S_{2}^{\mathrm{SDW}} & =\sum_{\mathbf{q}} B(\mathbf{q}) \mathbf{N}(-\mathbf{q}) \cdot \mathbf{N}(\mathbf{q}),
\end{aligned}
$$

with coefficients $A(\mathbf{p})=-2 V^{-1}-2 T \sum_{k} G\left(\mathbf{k}+\mathbf{p} / 2, \omega_{n}\right) \phi_{\Gamma}(\mathbf{k}+\mathbf{p} / 2) G(-\mathbf{k}+$ $\left.\mathbf{p} / 2,-\omega_{n}\right) \phi_{\Gamma}(-\mathbf{k}+\mathbf{p} / 2)$, and $B(\mathbf{q})=-2 J^{-1}(\mathbf{q})-2 T \sum_{k} G\left(\mathbf{k}, \omega_{n}\right) G\left(\mathbf{k}+\mathbf{q}, \omega_{n}\right)$. Here, $T$ is the temperature, $G\left(\mathbf{k}, \omega_{n}\right)=1 /\left(i \omega_{n}+\xi_{\mathbf{k}}\right)$ is the fermion propagator, $\omega_{n}$ is fermionic Matsubara frequency, and $\sum_{k}=\sum_{\mathbf{k}, \omega_{n}}$ is used to shorthand notation.

In what follows we make some standard assumptions. First, we assume that the saddle point TSC order parameter is dominated by the zero center of mass momentum component $\mathbf{D}_{0} \equiv \mathbf{D}(\mathbf{p}=0)$. Second, we assume that the saddle point SDW order parameter $\mathbf{N}$ is a real vector in r-space, and that it has Fourier components determined by Fermi surface nesting vectors $\mathbf{q}=\mathbf{Q}_{i}=\left( \pm Q_{a}, \pm Q_{b}, \pm Q_{c}\right)$ [5]. In this case, the coefficients $B\left(\mathbf{Q}_{i}\right)$ are identical for all $\mathbf{Q}_{i}$ 's, since the lattice dispersion in invariant under reflections and inversions compatible with the $D_{2 \mathrm{~h}}$ group. In addition, the coefficients of all higher order terms involving $\mathbf{N}\left(\mathbf{Q}_{i}\right)^{\prime} s$ share the same properties. Given that $\mathbf{N}(\mathbf{r})$ is real, and that we have periodic boundary conditions, we can choose a specific reference phase where $\mathbf{N}\left(\mathbf{Q}_{i}\right)$ are real and identical. Thus, we define $\mathbf{N}_{0} \equiv \mathbf{N}\left(\mathbf{Q}_{i}\right)$ for all $i$, and the quadratic terms are dominated in the long wavelength limit by $S_{2}^{\text {TSC }} \approx A(0)\left|\mathbf{D}_{0}\right|^{2}$ and $S_{2}^{\text {SDW }} \approx(m / 2) B\left(\mathbf{Q}_{1}\right)\left|\mathbf{N}_{0}\right|^{2}$, respectively. Here, $m$ is the number of nesting vectors, and $\mathbf{Q}_{1}=\left(Q_{a}, Q_{b}, Q_{c}\right)$ is chosen for definiteness.

Notice that the two order parameters $\mathbf{D}(\mathbf{p})$ and $\mathbf{N}(\mathbf{q})$ do not couple to quadratic order, because TSC and SDW are instabilities in particle-particle and particle-hole channels, respectively. Thus, the two orders are independent to this order, and their corresponding vector order parameters are free to rotate. However, this freedom is lost when fourth order terms are included. 
The coupling between $\mathbf{D}$ and $\mathbf{N}$ in fourth order is given by

$$
S_{4}^{\mathrm{C}}=\left(C_{1}+C_{2} / 2\right)\left|\mathbf{D}_{0}\right|^{2}\left|\mathbf{N}_{0}\right|^{2}-C_{2}\left|\mathbf{D}_{0} \cdot \mathbf{N}_{0}\right|^{2},
$$

where $C_{1}=m T \sum_{k} G\left(\mathbf{k}, \omega_{n}\right) G^{2}\left(-\mathbf{k},-\omega_{n}\right) G\left(-\mathbf{k}+\mathbf{Q}_{1},-\omega_{n}\right) \phi_{\Gamma}(-\mathbf{k}) \phi_{\Gamma}(\mathbf{k})$, and the other coefficient $C_{2}=m T \sum_{k} G\left(\mathbf{k}, \omega_{n}\right) G\left(\mathbf{k}+\mathbf{Q}_{1}, \omega_{n}\right) G\left(-\mathbf{k}-\mathbf{Q}_{1}, \omega_{n}\right) G\left(-\mathbf{k},-\omega_{n}\right) \phi_{\Gamma}\left(-\mathbf{k}-\mathbf{Q}_{1}\right) \phi_{\Gamma}(\mathbf{k})$. Notice that the second term on Eq. (8) can be parametrized as $-C_{2} \cos ^{2}(\theta)\left|\mathbf{D}_{0}\right|^{2}\left|\mathbf{N}_{0}\right|^{2}$, where $\cos ^{2} \theta \equiv\left|\mathbf{D}_{0} \cdot \mathbf{N}_{0}\right|^{2} /\left|\mathbf{D}_{0}\right|^{2}\left|\mathbf{N}_{0}\right|^{2} \leq 1$ is independent of $\left|\mathbf{D}_{0}\right|$ and $\left|\mathbf{N}_{0}\right|$. Since $\mathbf{D}_{0}$ is unitary, its global phase can be eliminated in $S_{4}^{\mathrm{C}}$, and $\theta$ can be regarded as the angle between $\mathbf{D}_{0}$ and $\mathbf{N}_{0}$. The coefficient $C_{2}$ for (TMTSF $)_{2} \mathrm{PF}_{6}$ is positive, indicating that $\mathbf{D}_{0}$ and $\mathbf{N}_{0}$ tend to be aligned $(\theta=0)$ or anti-aligned $(\theta=\pi)$.

Additional fourth order terms are

$$
S_{4}^{\mathrm{TSC}}=D_{1}\left|\mathbf{D}_{0}\right|^{4} ; \quad S_{4}^{\mathrm{SDW}}=D_{2}\left|\mathbf{N}_{0}\right|^{4}
$$

where $\quad D_{1} \quad=\quad T \sum_{k} G^{2}\left(\mathbf{k}, \omega_{n}\right) G^{2}\left(-\mathbf{k},-\omega_{n}\right) \phi_{\Gamma}^{2}(\mathbf{k}) \phi_{\Gamma}^{2}(-\mathbf{k}), \quad$ and $\quad D_{2} \quad=$ $(m / 2) T \sum_{k} G\left(\mathbf{k}, \omega_{n}\right) G^{2}\left(\mathbf{k}+\mathbf{Q}_{1}, \omega_{n}\right)\left[G\left(\mathbf{k}, \omega_{n}\right)+G\left(\mathbf{k}+2 \mathbf{Q}_{1}, \omega_{n}\right)\right]+(m / 4) T \sum_{k} G\left(\mathbf{k}, \omega_{n}\right) G(\mathbf{k}+$ $\left.\mathbf{Q}_{1}, \omega_{n}\right) \sum_{i}^{\prime} G\left(\mathbf{k}+\mathbf{Q}_{1}+\mathbf{Q}_{i}, \omega_{n}\right)\left[G\left(\mathbf{k}+\mathbf{Q}_{i}, \omega_{n}\right)+G\left(\mathbf{k}+\mathbf{Q}_{1}, \omega_{n}\right)\right]$. Here $\sum_{i}^{\prime}$ represents $\sum_{\mathbf{Q}_{i} \neq \pm \mathbf{Q}_{1}}$. This leads to the effective action

$$
S_{\text {eff }}=S_{0}+S_{2}+S_{4}
$$

where $S_{0}$ is the normal state contribution, $S_{2}=S_{2}^{\mathrm{TSC}}+S_{2}^{\mathrm{SDW}}$, and $S_{4}=D_{1}\left|\mathbf{D}_{0}\right|^{4}+D_{2}\left|\mathbf{N}_{0}\right|^{4}+$ $C(\theta)\left|\mathbf{D}_{0}\right|^{2}\left|\mathbf{N}_{0}\right|^{2}$ with $C(\theta)=C_{1}+C_{2} / 2-C_{2} \cos ^{2} \theta$. The phase diagram that emerges from this action leads to either bicritical or tetracritical points as illustrated in Fig. 1. When $R=C^{2}(0) /\left(4 D_{1} D_{2}\right)>1$ the critical point $\left(P_{c}, T_{c}\right)$ is bicritical and there is a first order transition line at $(m / 2) B\left(\mathbf{Q}_{1}\right)=A(0)$ when both $B\left(\mathbf{Q}_{1}\right)<0$ and $A(0)<0$, as seen in Fig. 1(a). However when $R<1,\left(P_{c}, T_{c}\right)$ is tetracritical and a coexistence region for TSC and SDW orders occurs when both $B\left(\mathbf{Q}_{1}\right)<0$ and $A(0)<0$, as seen in Fig. 1(b). The action $S_{\text {eff }}$ obtained in three dimensions is not $\mathrm{SO}(4)$ invariant, and $\mathrm{SO}(4)$ symmetry based theories [12] can only be applied to one-dimensional systems, but not to the highly anisotropic but three-dimensional Bechgaard salts.

The ratio $R \approx(0.12)^{2}<1$ for the Bechgaard salt $(\mathrm{TMTSF})_{2} \mathrm{PF}_{6}$ around $\left(P_{c}, T_{c}\right)$, when the interaction strengths $V, J$ are chosen to give the same $T_{c}=1.2 \mathrm{~K}$ at quarter filling for parameters $\left|t_{x}\right|=5800 \mathrm{~K},\left|t_{y}\right|=1226 \mathrm{~K},\left|t_{z}\right|=58 \mathrm{~K}$, used in combination with $\phi_{\Gamma}(\mathbf{k})=$ 
(a)

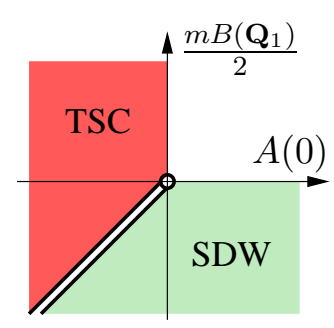

(b)

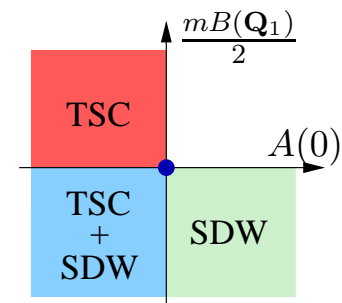

Normal
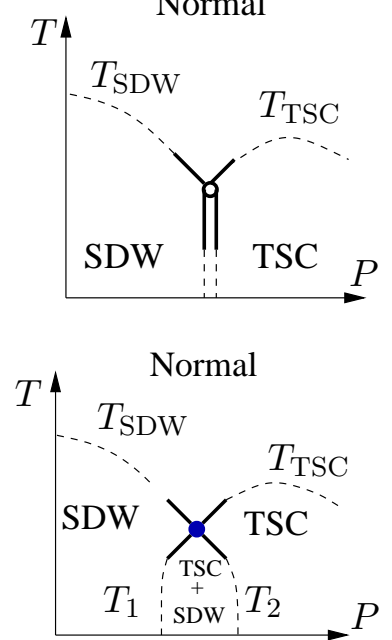

FIG. 1: $P-T$ phase diagrams indicating (a) first order transition line with no coexistence phase, and (b) two second order lines with coexistence region between TSC and SDW phases.

$\sin \left(k_{x} a\right)$ ( $p_{x}$-symmetry for TSC) and the nesting vectors $\mathbf{Q}=( \pm \pi / 2 a, \pm \pi / 2 b, 0)(m=$ 4). This shows that (TMTSF) ${ }_{2} \mathrm{PF}_{6}$ has an TSC/SDW coexistence region as suggested by experiments [9, 10, 11].

To investigate the TSC/SDW coexistence region the effective action (10) [with $\mathbf{Q}=$ $\left.\left( \pm Q_{a}, \pm Q_{b}, 0\right)\right]$ is Fourier transformed into real space to give the Ginzburg-Laudau (GL) free energy density

$$
\mathcal{F}=\mathcal{F}_{n}+\mathcal{F}_{\mathrm{TSC}}+\mathcal{F}_{\mathrm{SDW}}+\mathcal{F}_{\mathrm{C}}
$$

where $\mathcal{F}_{n}$ is the normal state contribution, and $\mathcal{F}_{\mathrm{C}}=C(\theta)|\mathbf{N}(\mathbf{r})|^{2}|\mathbf{D}(\mathbf{r})|^{2}$ is the coupling term of the two order parameters. The TSC contribution is $\mathcal{F}_{\mathrm{TSC}}=A(0)|\mathbf{D}(\mathbf{r})|^{2}+D_{1}|\mathbf{D}(\mathbf{r})|^{4}+$ $\sum_{i j} \gamma_{\text {TSC }}^{i j}\left[\partial_{i} \mathbf{D}(\mathbf{r})\right] \cdot\left[\partial_{j} \mathbf{D}(\mathbf{r})\right]$, where $\gamma_{\text {TSC }}^{i j}$ is obtained from a small $\mathbf{p}$ expansion of $A(\mathbf{p})$. The SDW contribution is $\mathcal{F}_{\mathrm{SDW}}=\int d \mathbf{r}^{\prime}\left[B\left(\mathbf{r}, \mathbf{r}^{\prime}\right) \mathbf{N}(\mathbf{r}) \cdot \mathbf{N}\left(\mathbf{r}^{\prime}\right)\right]+\left(D_{2} / m^{2}\right)|\mathbf{N}(\mathbf{r})|^{4}$, where $B\left(\mathbf{r}, \mathbf{r}^{\prime}\right)$ is the Fourier transform of $B(\mathbf{q})$ in Eq. (7). For the Bechgaard salt parameters, the prefactor $C(0)$ of the coupling term $\mathcal{F}_{\mathrm{C}}$ is positive, and hence represents a local repulsive interaction between the TSC and SDW order parameters. As a consequence, the TSC order parameter is non-uniform in the TSC/SDW coexistence region, and has a modulation induced by the SDW order parameter. Since $R \ll 1$ for (TMTSF $)_{2} \mathrm{PF}_{6}$, the coupling term $\mathcal{F}_{\mathrm{C}}$ is small in comparison with the other fourth order coefficients $D_{1}, D_{2}$, and a perturbative solution is possible for $|\mathbf{D}(\mathbf{r})|$ and $|\mathbf{N}(\mathbf{r})|$. At assumed zero TSC/SDW coupling $C(0)=0$, the 

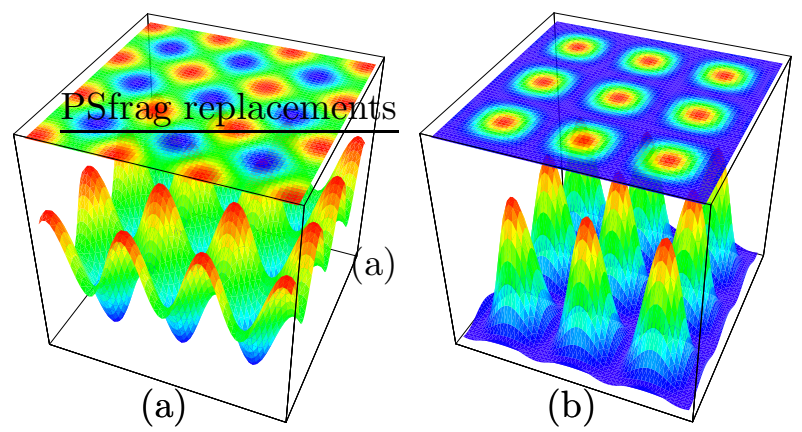

FIG. 2: Magnitude square of (a) TSC and (b) SDW order parameters in the coexistence region.

saddle point modulation for the SDW order parameter is $\mathbf{N}(\mathbf{r})=m \mathbf{N}_{0} \cos \left(\mathbf{Q}_{1} \cdot \mathbf{r}\right)$, with $\left|\mathbf{N}_{0}\right|=\left[-m B\left(\mathbf{Q}_{1}\right) / 3 D_{2}\right]^{1 / 2}$, while the saddle point for the TSC order parameter is $\mathbf{D}(\mathbf{r})=\mathbf{D}_{0}$ with $\left|\mathbf{D}_{0}\right|=\left[-A(0) / 2 D_{1}\right]^{1 / 2}$. Including the coupling $\mathcal{F}_{\mathrm{C}}$ the new solution for the magnitude of TSC order parameter is

$$
\begin{aligned}
|\mathbf{D}(\mathbf{r})|-\left|\mathbf{D}_{0}\right| & =-v \frac{\left|N_{0}\right|^{2}}{\left|D_{0}\right|} R^{1 / 2}\left[\frac{\cos \left(2 Q_{a} x\right)}{4+8 \xi_{x}^{2} Q_{a}^{2}}+\frac{\cos \left(2 Q_{b} y\right)}{4+8 \xi_{y}^{2} Q_{b}^{2}}\right. \\
& \left.+\frac{\cos \left(2 Q_{a} x\right) \cos \left(2 Q_{b} y\right)}{4+8 \xi_{x}^{2} Q_{a}^{2}+8 \xi_{y}^{2} Q_{b}^{2}}+\frac{1}{4}\right]
\end{aligned}
$$

which shows explicitly $2 Q_{a}$ and $2 Q_{b}$ modulations along the a and $\mathbf{b}^{\prime}$ axes, respectively. Here, $\xi_{i}=\sqrt{\left|\gamma_{\text {TSC }}^{i i} / A(0)\right|}$ represents the TSC coherence length along the $i$ direction, and $v=\left(6 D_{2} / D_{1}\right)^{1 / 2}$. Notice that the modulation in $|\mathbf{D}(\mathbf{r})|$ disappears as the SDW order goes away $\left|\mathbf{N}_{0}\right| \rightarrow 0$. The qualitative behavior of $|\mathbf{D}(\mathbf{r})|$ is shown in Fig. 2(a). The new solution for the SDW order parameter to the first order correction is $|\mathbf{N}(\mathbf{r})|=\sum_{i}(1-$ $\left.R^{1 / 2}\left|\mathbf{D}_{0}\right|^{2} / 4 v\left|\mathbf{N}_{0}\right|^{2}\right)\left|\mathbf{N}_{0}\right| \cos \left(\mathbf{Q}_{i} \cdot \mathbf{r}\right)$, and can be seen in Fig. 2(b). Notice that the maxima of $|\mathbf{D}(\mathbf{r})|$ coincide with the minima of $|\mathbf{N}(\mathbf{r})|$ indicating that the TSC and SDW orders try to be locally excluded. Since the TSC and SDW modulations are out of phase, experiments that are sensitive to the spatial distribution of the spin density or Cooper pair charge density may reveal the coexistence of these inhomogeneous phases.

Next, we analyze the effect of magnetic fields on this coexistence region. A uniform magnetic field $\mathbf{H}$ couples with charge via the Peierls substitution $\mathbf{k} \rightarrow \mathbf{k}-|e| \mathbf{A}$ in the dispersion relation given in Eq. (11), where $\mathbf{A}$ is the vector potential, and couples with spin via the paramagnetic term $\mathcal{H}_{\mathrm{P}}=-\mu_{0} \mathbf{H} \cdot \sum_{\mathbf{k}, \alpha \beta} c_{\mathbf{k} \alpha}^{\dagger} \boldsymbol{\sigma}_{\alpha \beta} c_{\mathbf{k} \beta}$, where $\mu_{0}$ is the effective magnetic 
moment. Thus, the effective Hamiltonian becomes

$$
\mathcal{H}_{\mathrm{eff}}=\mathcal{H}_{0}(\mathbf{k} \rightarrow \mathbf{k}-|e| \mathbf{A})+\mathcal{H}_{\mathrm{TSC}}+\mathcal{H}_{\mathrm{SDW}}+\mathcal{H}_{\mathrm{P}}
$$

Upon integration of the fermions, the corresponding effective action is

$$
S_{\text {eff }}(\mathbf{H})=S_{0}(\mathbf{H})+S_{2}(\mathbf{H})+S_{4}(\mathbf{H})
$$

where $S_{0}(\mathbf{H})=S_{0}+|\mathbf{H}|^{2} / 8 \pi-\chi_{n}|\mathbf{H}|^{2} / 2, \quad \chi_{n}$ is the uniform electronic spin susceptibility of the normal state, $S_{2}(\mathbf{H})$ is obtained from $S_{2}$ by the Peierls substitution, and $S_{4}(\mathbf{H})=S_{4}+\left(E_{1}+E_{2} / 2\right)|\mathbf{H}|^{2}\left|\mathbf{D}_{0}\right|^{2}-E_{2}\left|\mathbf{H} \cdot \mathbf{D}_{0}\right|^{2}+\left(F_{1}-F_{2} / 2\right)|\mathbf{H}|^{2}\left|\mathbf{N}_{0}\right|^{2}+F_{2} \mid \mathbf{H}$. $\left.\mathbf{N}_{0}\right|^{2}$. The coefficients are $E_{1}=2 \mu_{0}^{2} T \sum_{k} G^{3}\left(-\mathbf{k}, \omega_{n}\right) G\left(\mathbf{k},-\omega_{n}\right) \phi_{\Gamma}(\mathbf{k}) \phi_{\Gamma}(-\mathbf{k}), \quad E_{2}=$ $2 \mu_{0}^{2} T \sum_{k} G^{2}\left(\mathbf{k}, \omega_{n}\right) G^{2}\left(-\mathbf{k},-\omega_{n}\right) \phi_{\Gamma}(\mathbf{k}) \phi_{\Gamma}(-\mathbf{k}), F_{1}=m \mu_{0}^{2} T \sum_{k} G^{3}\left(\mathbf{k}, \omega_{n}\right)\left[G\left(\mathbf{k}+\mathbf{Q}_{1}, \omega_{n}\right)+\right.$ $\left.G\left(\mathbf{k}-\mathbf{Q}_{1}, \omega_{n}\right)\right]$, and $F_{2}=m \mu_{0}^{2} T \sum_{k} G^{2}\left(\mathbf{k}, \omega_{n}\right) G^{2}\left(\mathbf{k}+\mathbf{Q}_{1}, \omega_{n}\right)$. A detailed calculation shows that the coefficient $E_{1}=-E_{2} / 2$, hence the coupling of $\mathbf{H}$ to $\mathbf{D}$ can be described in the more familiar form $F_{M}-\sum_{\mu \nu} H_{\mu} \chi_{\mu \nu} H_{\nu} / 2$, where $\chi_{\mu \nu}=\chi_{n} \delta_{\mu \nu}+E_{2} D_{\mu}^{*} D_{\nu}$.

For Bechgaard salts, the coefficients $E_{2}<0$ and $F_{2}>0$ indicating that $\mathbf{D}$ and $\mathbf{N}$ prefer to be perpendicular to the magnetic field $\mathbf{H}$. These conditions, when combined with $C_{2}>0$ in Eq. (8), indicate that $\mathbf{D}$ and $\mathbf{N}$ prefer to be parallel to each other, but perpendicular to $\mathbf{H}$. However, the relative orientation of these vectors in small fields is affected by spin anisotropy effects which were already observed in (TMTSF) ${ }_{2} \mathrm{PF}_{6}$, where the easy axis for $\mathbf{N}$ is the $\mathbf{b}^{\prime}$ direction [6]. Such an anisotropy effect can be described by adding a quadratic term $-u_{N} N_{b^{\prime}}^{2}$ with $u_{N}>0$, which favors $\mathbf{N} \| \mathbf{b}^{\prime}$. Similarly, the $\mathbf{D}$ vector also has anisotropic effect caused by spin-orbit coupling, and can be described by adding a quadratic term $-u_{D} D_{i}^{2}$, where $i$ is the easy axis for TSC. (Quartic TSC and SDW terms also become anisotropic.)

However, a sufficiently large $\mathbf{H} \| \mathbf{b}^{\prime}$ can overcome spin anisotropy effects, and drive the $\mathbf{N}$ vector to flop onto the $\mathbf{a}-\mathbf{c}^{*}$ plane. This canting (flop) transition was reported [6] in $(\mathrm{TMTSF})_{2} \mathrm{PF}_{6}$ for $H \approx 1 \mathrm{~T}$ at zero pressure and $T=8 \mathrm{~K}$. If such a spin-flop transition persists near the TSC/SDW critical point $\left(P_{c}, T_{c}\right)$ as suggested in our discussion, then the flop transition of the $\mathbf{N}$ vector forces the $\mathbf{D}$ vector to flop as well, and has potentially serious consequences to the superconducting state. Schematic phase diagrams are are shown in Fig. 3(a) and 3(b). For $P<P_{c}$, if a flop transition occurs for $H_{F}<H_{1}(0)$ [see Fig. 3(a)], then $\mathbf{N}$ flops both in the pure SDW and in the TSC/SDW coexistence phases, in which case it forces $\mathbf{D}$ vector to flop as well. If the flop transition occurs for $H_{\mathrm{SDW}}(0)<H_{F}<H_{1}(0)$ (not 

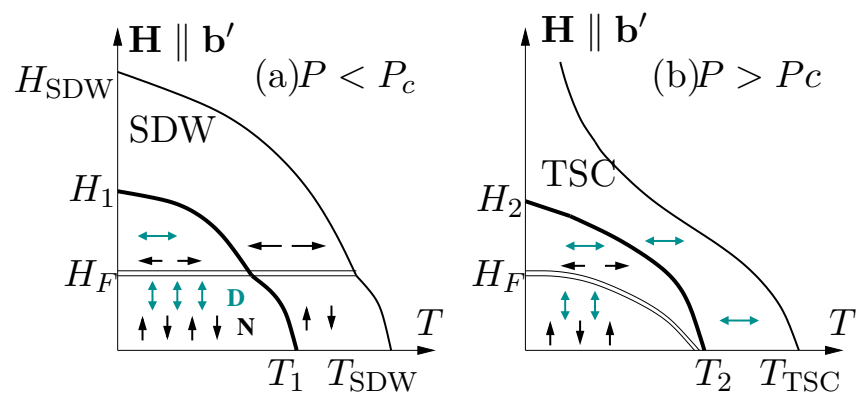

FIG. 3: $H-T$ phase diagrams showing the TSC/SDW coexistence region (thick solid line) and canting transitions (double line) for (a) $P<P_{c}$ and (b) $P>P_{c}$.

shown) then only the pure SDW phase is affected. This situation is qualitatively different for $P>P_{c}$. In the zero (weak) spin-orbit coupling limit the $\mathbf{D}$ vector is free to rotate in a magnetic field and tends to be perpendicular to $\mathbf{H}$ in order to minimize its magnetic free energy $F_{M}$. Thus, for $\mathbf{H} \| \mathbf{b}^{\prime}$ and $|\mathbf{H}|>H_{2}$, the $\mathbf{D}$ vector lies in the a-c* plane since there is no SDW order. However, at lower temperatures and small magnetic fields when TSC and SDW orders coexist, the spin anisotropy field forces $\mathbf{N}$ to be along $\mathbf{b}^{r}$ / and $\mathbf{N}$ forces $\mathbf{D}$ to flop from the a-c* plane to $\mathbf{b}^{\prime}$ direction. This canting transition occurs at $H_{F}<H_{2}(0)$ [see Fig. 3(b)], when $\mathbf{N}$ flops in the TSC/SDW coexistence phase, and forces the $\mathbf{D}$ vector to flop as well.

In summary, we showed that the TSC and SDW order parameters can coexist in the $P-T$ phase diagram of quasi-one-dimensional organic conductors. In the coexistence region the TSC order parameter is non-uniform, and its modulation is induced via the SDW order parameter. We also showed that theories based on $\mathrm{SO}(4)$ symmetry cannot be applied to these highly anisotropic three-dimensional systems, since they are strictly valid only in the one-dimensional limit. Furthermore, we discussed qualitatively magnetic field effects on the coexistence region. We proposed that a magnetic field induced canting transition of the SDW order parameter affects dramatically the phase diagram of the coexistence region, both below and above the critical pressure. We would like to thank NSF for support (DMR-0304380).

[1] E. Dagotto, Rev. Mod. Phys. 66, 763 (1994).

[2] Y. Maeno et. al., Nature 372, 532 (1994). 
[3] C. Pfleiderer et al., Nature 412, 58 (2001).

[4] S. S. Saxena et al., Nature 406, 587 (2000).

[5] T. Ishiguro, K. Yamaji, and G. Saito, organic superconductors (Springer, Berlin, 1998), 2nd ed.

[6] K. Mortensen et. al., Phys. Rev. Lett. 46, 1234 (1981).

[7] I. J. Lee et. al., Phys. Rev. Lett. 78, 3555 (1997).

[8] I. J. Lee et. al., Phys. Rev. B 68, 092510 (2003).

[9] T. Vuletić et. al., Eur. Phys. J. B 25, 319 (2002).

[10] A. V. Kornilov et. al., Phys. Rev. B 69, 224404 (2004).

[11] I. J. Lee et. al., Phys. Rev. Lett. 94, 197001 (2005).

[12] D. Podolsky et. al., Phys. Rev. Lett. 93, 246402 (2004).

[13] W. Zhang and C. A. R. Sá de Melo, J. Appl. Phys. 95, 10B108 (2005).

[14] R. D. Duncan, C. D. Vaccarella, and C. A. R. Sá de Melo, Phys. Rev. B 64, 172503 (2001); R. D. Duncan, R. W. Cherng, and C. A. R. Sá de Melo, Physica C 391, 98 (2003). 